\title{
ECG SIGNAL COMPRESSION USING COMBINED MODIFIED DISCRETE-COSINE AND DISCRETE-WAVELET TRANSFORMS
}

\author{
Sabah M. Ahmed \\ Assistant Professor, Department of Electrical and Electronics \\ Engineering, Faculty of Engineering, Assiut University , Assiut, Egypt \\ (sabahma@yahoo.com).
}

(Received October 9, 2005 Accepted November 10, 2005)

\begin{abstract}
A new hybrid two-stage electrocardiogram (ECG) signals compression method based on the Modified Discrete Cosine Transform (MDCT) and Discrete Wavelet Transform (DWT) is proposed. The ECG signal is partitioned in blocks and the MDCT is applied to each block to decorrelate the spectral information. Then, the DWT is applied to each MDCT block of the signal. Removing spectral redundancy is achieved by compressing the subordinate components more than the dominant components. The resulting wavelet coefficients are then threshold and compressed using energy packing and binary-significant map coding technique for storage space saving. Experimenting on an ECG records from the MIT-BIH database is performed with various combinations of the MDCT and wavelet filters at different transformation levels, and quantization intervals. The decompressed signals are evaluated using percentage root mean square error (PRD) and zero-mean root mean square error $\left(P R D_{1}\right)$ measures. The results showed that the proposed method provides low bit-rate and high quality of the reconstructed signal. It offers a compressed ratio $(C R)$ in between 12.6 and 21.5 average PRD of $5.89 \%$, which would be suitable for most monitoring and diagnoses applications. Experiments with ECG signals used in results from the literature showed that the proposed method compares favorably with various state-of-the-art ECG compressors.
\end{abstract}

KEYWORDS: Data compression; Electrocardiogram; Wavelet transform; Discrete cosine transform; Energy packing; Binary-significant map coding.

\section{INTRODUCTION}

As a diagnosis tool of cardiac diseases, the ECG is a very important physiological signal. It provides essential information to cardiologist that is used for both monitoring and diagnostic purposes. However, the amount of ECG data grows with the increase of sampling rate, sample resolution, recording time, and the number of channels. This gradually becomes a problem when storage space and bandwidth are very limited. Uncompressed ECG data requires considerable storage capacity and transmission 
bandwidth. In most applications, the sampling rate varies from 125 to $500 \mathrm{~Hz}$ and 8,12 or 16 bits may represent each data sample. This will lead to an accumulation of ECG data ranging from $60 \mathrm{Kbits}$ per minute to $480 \mathrm{Kbits}$ per minute. Despite rapid progress in mass-storage density, processor speeds, and digital communication system performance, demand for data storage capacity and data-transmission bandwidth continues to outstrip the capabilities of available technologies [1].

A typical computerized medical signal processing system acquires a large amount of data that is difficult to store and transmit. It is very desirable to find a method of reducing the quantity of data without loss of important information. Under these circumstances, preserving the most useful information when compressing a signal to an acceptable size becomes the central goal of ECG data compression techniques proposed in literature over the past 30 years [2]. Conventional ECG signal compression techniques explore useful information within the signal. A lossless compression is the best choice as long as the compression ratio is acceptable, but it usually cannot offer a satisfactory CR. This type of ECG compression techniques hardly achieves a compression ratio greater than 2. To obtain significant signal compression, lossy compression is preferable to a lossless compression [3]. In this case, compression is accomplished by applying an invertible orthogonal transform to the signal, and one tries to reduce the redundancy present in the new representation. Due to its decorrelation and energy compaction properties and to the existence of efficient algorithms to compute it, discrete cosine transform [4], and modified discrete cosine transform [5]-[7], have been widely investigated for ECG signal compression. Over the years, a variety of other linear transforms have been developed which include DFT, DWT and many more, each with its own advantages and disadvantages [8]. Among these techniques, DWT has been proven to be very efficient for ECG signal coding [3], [9]. Compared to other methods, DWT has gained widespread acceptance in signal processing in general, and in ECG compression research in particular. In this case, wavelet-based schemes outperform other coding schemes like the one based on DFT [1], [10].

In this paper, a hybrid two-stage ECG signals compression method based on MDCT and DWT is proposed. Their combination removes the spectral redundancy by compressing the subordinate components more than the dominant components. The resulting transformed coefficients that represent the transformational signal are then threshold and compressed using a new coding technique for storage space saving. Experimenting on an ECG records from the MIT-BIH database is performed with various combinations of MDCT and DWT at different transformation levels, and quantization intervals [11].

\section{WAVELET TRANSFORMATION}

Wavelets are functions defined over a finite interval and having an average value of zero [8]. The basic idea of the wavelet transform is to represent any arbitrary function $\mathrm{x}(\mathrm{t})$ as a superposition of a set of basis functions (wavelets). These basis functions or baby wavelets are obtained from a single prototype mother wavelet, by dilations or contractions (scaling) and translations (shifts). So, a wavelet transform decomposes a signal into a series of smooth signals and associated detailed signals at different 
resolution levels [12]. At each level, the smooth signal and associated detailed signal have all of the information necessary to reconstruct the smooth signal at the next higher resolution level. A multi-resolution WT involves two functions: a mother wavelet $\psi(\mathrm{t})$ and a scaling function $\phi(t)$. Since 1989, techniques based on filter banks concept have been proposed by various researchers under the name of Wavelet Coding (WC) using filters specifically designed for this purpose [8]-[10]. In [8], Daubechies was the first to discover that the discrete-time filters or quadrature mirror filter (QMF) banks can be iterated and under certain regularity conditions will lead to continuous-time wavelets. This is a very practical and extremely useful wavelet decomposition scheme, since FIR discrete-time filters can be used to implement them. It follows that the orthonormal bases in [8] correspond to a subband-coding scheme with exact reconstruction property, using the same FIR filters for reconstruction as for decomposition. Wavelets did not gain popularity in ECG coding until Daubechies established this link in late 1980s [8]. Later a systematic way of constructing a family of compactly supported biorthogonal wavelets was developed by Cohen, Daubechies, and Feauveau (CDF) [12].

\section{MODIFIED DISCRETE COSINE TRANSFORM}

The discrete cosine transform (DCT) is a Fourier-related transform of roughly twice the length, operating on real data with even symmetry, where in some variants the input and/or output data are shifted by half a sample. MDCT is a linear orthogonal lapped transform, based on the idea of time domain aliasing cancellation (TDAC) and designed to be performed on consecutive blocks of a larger dataset, where subsequent blocks are overlapped so that the last half of one block coincides with the first half of the next block. This overlapping, in addition to the energy-compaction qualities of the DCT, makes the MDCT especially attractive for signal compression applications. Thus, it helps to avoid artifacts stemming from the block boundaries [5]-[6]. MDCT is critically sampled, which means that though it is 50\% overlapped, a sequence data after MDCT has the same number of coefficients as samples before the transform (after overlap-and-add). This means that, a single block of IMDCT data does not correspond to the original block on which the MDCT was performed. When subsequent blocks of inverse transformed data are added, the errors introduced by the transform cancel out TDAC. The MDCT is defined as [6]:

$$
X_{C}(k)=\sum_{n=0}^{N-1} x(n) \cos \left[\left(n+\frac{M+1}{2}\right)\left(k+\frac{1}{2}\right) \frac{\pi}{M}\right], \quad k=0,1, \ldots M-1
$$

where, $x(n), n=0,1,2, \ldots, N-1$ is the sequence to be transformed, $\mathrm{N}=2 \mathrm{M}$ is the window length and $\mathrm{M}$ is the number of transform coefficients. The computation burden can be reduced if the transform coefficients given by equation (1) are rewritten in the following recursive form

Where,

$$
X_{C}(k)=x(0) \cos \left[(M+1) \frac{\theta_{k}}{2}\right]+V_{1} \cos \left[(M+3) \frac{\theta_{k}}{2}\right]-V_{2} \cos \left[(M+1) \frac{\theta_{k}}{2}\right]
$$

$$
V_{m}=x(m)+2 \cos \theta_{k} V_{m+1}-V_{m+2}, m=N-1, N-2, \ldots, 1,0
$$


And $\quad \theta_{k}=\left(k+\frac{1}{2}\right) \frac{\pi}{M}$

The MDCT computation algorithm of a data sequence $x(n)$ can be summarized in the following:

1. Partition the data sequence in $\mathrm{N}_{\mathrm{b}}$ consecutive blocks, each one with $N=64$ samples.

2. Recursively generate the $V_{m}$ from the input sequence $x(n)$ according to (3) and (4).

3. Calculate the MDCT coefficients for each block by evaluating the k-th MDCT coefficient using (2) at the $\mathrm{N}$-th step.

The inverse MDCT is known as the IMDCT. Because there are different numbers of inputs and outputs, at first glance it might seem that the MDCT should not be invertible. However, perfect invertability is achieved by adding the overlapped IMDCTs of subsequent overlapping blocks, causing the errors to cancel and the original data to be retrieved. The IMDCT transforms the $M$ real coefficients, $X_{C}(0)$, $X_{C}(1), \ldots, X_{C}(M-1)$, into $N=2 M$ real numbers, $x(0), x(1), \ldots, x(N-1)$, according to the formula:

$$
x(n)=\sum_{k=0}^{M-1} X_{C}(k) \cos \left[\left(n+\frac{M+1}{2}\right)\left(k+\frac{1}{2}\right) \frac{\pi}{M}\right], \quad n=0,1, \ldots N-1
$$

Again, the computation burden of $x(n)$ can be reduced considerably if equation (5) is rewritten in the following recursive form

$$
x(n)=X_{C}(0) \cos \left[\frac{\theta_{n}}{2}\right]+V_{1} \cos \left[\frac{3 \theta_{n}}{2}\right]-V_{2} \cos \left[\frac{\theta_{n}}{2}\right]
$$

Where, $V_{m}=X_{C}(m)+2 \cos \theta_{n} V_{m+1}-V_{m+2} \quad$ and $\quad \theta_{n}=\left(n+\frac{M+1}{2}\right) \frac{\pi}{M}$.

\section{COMPRESSION AND DISTORTION MEASURES}

All data compression algorithms seek to minimize data storage by eliminating redundancy where possible by increasing the compression ratio. It is defined as the ratio of the number of bits of the original signal to the number required to store the compressed signal. A high compression ratio is wanted, typically, but using this alone to compare data compression algorithms is not acceptable. Generally the bandwidth, sampling frequency, and precision of the original data very much affect the compression ratio. A data compression algorithm must also represent the data with acceptable fidelity.

In ECG signal compression, the clinical acceptability of the reconstructed signal has to be determined through visual inspection by cardiologists. The error signal resulting from the difference between the reconstructed signal and the original one may also be measured by numerical measure. A lossless data compression algorithm produces zero residual, and the reconstructed signal exactly replicates the original signal. However, clinically acceptable quality is neither guaranteed by a low nonzero residual nor ruled out by a high numerical residual. The criterion for testing compression algorithms performance includes three components: compression measure, reconstruction error 
and computational complexity. The compression measure and the reconstruction error are usually dependent on each other and are used to create the rate-distortion function of the algorithm. The computational complexity component is part of the practical implementation consideration.

\subsection{Distortion Measures}

One of the most difficult problems in ECG compression and reconstruction is defining the error criterion. The purpose of the compression system is to remove the irrelevant information which does not contain diagnostic information. Consequently, the error criterion has to be defined such that it will measure the ability of the reconstructed signal to preserve the relevant information. As yet, there is no such mathematical structure to this criterion, and all accepted error measures are still variations of the Mean Square Error or absolute error, which are easy to compute mathematically, but are not always diagnostically relevant. In technical literature, the distortion resulting from the ECG processing is frequently measured by the percent root-mean-square difference (PRD) [1]-[4]. It is most commonly defined as:

$$
P R D=\sqrt{\frac{\sum_{n=1}^{N}(x(n)-\tilde{x}(n))^{2}}{\sum_{n=1}^{N} x^{2}(n)}} \times 100
$$

where, $x(n)$ and $\tilde{x}(n)$ are the values of the original and reconstructed samples, respectively, and $N$ is the length of the window over which the PRD is calculated. Another definition, called here $\mathrm{PRD}_{1}$, subtracts from the signal its average value $\bar{x}$, in the denominator of the above equation.

$$
P R D_{1}=\sqrt{\frac{\sum_{n=1}^{N}(x(n)-\tilde{x}(n))^{2}}{\sum_{n=1}^{N}(x(n)-\bar{x})^{2}}} \times 100
$$

This definition is independent in the DC level of the original signal. One can use the first definition, but the original signal has to have a zero mean. Despite their wide use, $\mathrm{PRD}$ and $\mathrm{PRD}_{1}$ do not indicate precisely the quality of signal's reconstruction [9] and the decompressed signal has also to be evaluated by visual inspection.

\subsection{Compression Measures}

The size of compression is often measured by CR, which is defined as the ratio between the bit rate of the original signal $\left(b_{\text {original }}\right)$ and the bit rate of the compressed one $\left(b_{\text {compressed }}\right)$. 


$$
C R=\frac{b_{\text {original }}}{b_{\text {compressed }}}
$$

The problem of using the above definition of CR is that every algorithm is fed with an ECG signal that has a different sampling frequency and a different number of quantization levels; thus, the bit rate of the original signal is not standard. Some attempts were made in the past to define standards for sampling frequency and quantization, but these standards were not implemented and the algorithms' developers still use rates and quantizers that are convenient to them [2]. In the literature [9], some authors use the number of bits transmitted per sample of the compressed signal as a measure of information rate. This measure removes the dependency on the quantizer resolution, but the dependence on the sampling frequency remains. Another way is using the number of bits transmitted per second [13]. This measure removes the dependence on the quantizer resolution as well as the dependence on the sampling frequency.

\section{QUANTIZATION AND CODING ALGORITHMS}

A quantizer simply reduces the number of bits needed to store the transformed coefficients by reducing the precision of those values. Since this is a many-to-one mapping, it is a lossy process and is the main source of compression in an encoder. A quantization scheme maps a large number of input values into a smaller set of output values. This implies that some information is lost during the quantization process. The original wavelet coefficients $c(n)$ cannot be recovered exactly after quantization. An encoder further compresses the quantized values losslessly to give better overall compression. The most commonly used encoders are the Huffman encoder and the arithmetic encoder, although for applications requiring fast execution, simple runlength encoding (RLE) has proven very effective [10]. In the following, wavelet coefficients quantization and coding algorithms are described.

\subsection{Energy Packing Efficiency Strategy}

In this section, the quantization strategy adopted is based on the energy packing efficiency (EPE). It guarantees the balance between the compression achievement and information loss. Here, quantization process is performed by selecting an appropriate threshold level $\lambda$ to control the compression ratio. Due to the careful representation by combining the MDCT and DWT, it is reasonable to assume that only a few coefficients contain information about the real signal while others appear as less important details. The goal is to extract these significant coefficients and to ignore others smaller than $\lambda$. The optimal value of $\lambda$ is determined such that the reconstructed signal is as close to the original one as possible. Usually the selection of optimal threshold level is not an easy task, because some of the coefficients that represent the actual signal details may be also killed, and as a result, signal distortion is the side effect [17].

As it can be deduced from the above discussion, the approximation band is the smallest band in size and it includes high amplitude approximation coefficients. The wavelet coefficients other than these included by the approximation band, detail coefficients, 
have small magnitudes. Most of the energy is captured by these coefficients of the lowest resolution band. This can be seen from the decomposition of 4096-sample ECG signal up to the fifth level. The total energy of the signal is 94393.5 . About $99.73 \%$ of this energy is concentrated in the 136 approximation coefficients and only $0.27 \%$ of the energy is concentrated in the remaining 3960 detail coefficients. Here, threshold levels are defined according to the energy packing efficiencies of the signal for all subbands. EPE for a set of coefficients in the ith subband is defined as the ratio of the energy captured by the subband coefficients and the energy captured by the whole number of coefficients.

$$
E P E_{i}=\frac{\sum_{n=1}^{L_{i}}(c(n))^{2}}{\sum_{n=1}^{L}(c(n))^{2}} x 100
$$

Where $L_{i}$ and $L$ are the number of coefficients in the ith subband and the whole number of coefficients respectively. A large threshold could attain high data reduction but poor signal fidelity and a small threshold would produce low data reduction but high signal fidelity. To explore the effect of threshold level $(\lambda)$ selection and the coefficients representation on the compression ratio and PRD, the following thresholding rule is set:

Keep all the wavelet coefficients in the approximation subband without thresholding and calculate the threshold value for each details subband separately by preserving the highest amplitude wavelet coefficients in the ith details subband that contribute to $\alpha_{i} \%$ of the energy in that subband.

One important feature of this rule is that the integer part of the wavelet coefficients in each subband is represented by different number of bits.

\subsection{Binary Significant Map Coding Algorithm}

The coding algorithm adopted here is based on grouping the significant coefficients in one vector and the locations of the insignificant coefficients in another vector. The significant coefficients are arranged from high scale coefficients to low scale coefficients. Each significant coefficient is decomposed into integer part and fractional part, where M-bits are assigned to represent the integer part (signed representation) and $\mathrm{N}$-bits represent the fractional part; i.e. each coefficient is represented by $\mathrm{N}+\mathrm{M}$ bits. A binary significant map is used as flags to indicate if the coefficient is significant or not. This binary stream is compressed further as will be shown in the following:

1. Threshold the wavelet coefficients, $c(n)$, to produce the threshold coefficients $\bar{c}(n)$. The threshold level $(\lambda)$ is determined by using the above-mentioned rule such that the distortion in the reconstructed signal $\bar{x}_{i}$ is acceptable. The distortion is measured using PRD and/or visual inspection. The optimal non-orthogonal wavelet transform developed in [5] may be used to minimize the PRD in least mean square sense. 
2. Search the vector $\bar{c}(n)$ to isolate the significant coefficient in another vector $\hat{C}_{S}(m)$.

3. Use finite word length representation to represent the integer and fractional parts of the coefficients, $\hat{C}_{S}(m)$. The number of bits used to represent these coefficients is determined as follows:

3.1 Search the vector $\hat{C}_{S}(m)$ to find the maximum coefficient (in absolute value) and determine the number of bits that represents this coefficient. This can be done by finding $k=\operatorname{Int}\left\langle\max \left|\hat{C}_{S}(m)\right|\right\rangle$ where $\operatorname{Int}\langle$.$\rangle denotes$ the integer part. Then convert $\mathrm{k}$ to a binary number and count the number of bits, M.

3.2 Similarly, find the number of bits, $\mathrm{N}$, that represent the minimum value of the fractional part of each significant coefficient in such a way to keep the distortion within acceptable limits.

4. Generate a binary stream, $b(n)$, of 1's and 0 's that encodes the zero-locations in $\bar{c}(n)$. This is done by coding each significant coefficient in $\bar{c}(n)$ by a binary 1 . The length of the binary stream equals $\mathrm{n}_{1}$, where $\mathrm{n}_{1}$ designates the index value of the last significant coefficients in $\bar{c}(n)$. Hence, there is no need to encode the zeros for $n>n_{1}$. The value of $n_{1}$ need not be stored because it can be determined as the length of the vector $b(n)$ in the decoding process.

5. Compress the binary stream using run length encoding of 0 's and 1's. This is done as follows:

5.1 Set $\mathrm{i}=1$, Run-type $=\mathrm{b}(\mathrm{i})$, and set the run length $\mathrm{Z}$ to 1 ;

5.2 If $b(i) \neq b(i+1)$ increment $i$ by $Z$. Else, while $b(i+1)=b(i)$, increment $i$ by 1 and $\mathrm{Z}$ by 1 end; end.

5.3 From Table (1), find the inequality that $Z$ satisfies. Then output the symbol that specifies the run type followed by the number Z. i.e., $\operatorname{code}=[\operatorname{code} \chi Z$ ] , where $\chi$ designates concatenation operator.

5.4 If index $<\mathrm{n}_{1}$ set $\mathrm{Z}=1$ and go to step (5.1).

6. Represent the obtained run length code in binary format. There are 16 different symbols that can be generated from step 5. These are the digits 0-9 and the letters A-F. Hence, 4 bits can be used to represent each symbol.

Table (1): Run Length Encoding of 0's and 1's.

\begin{tabular}{|c|c|c|}
\hline Symbol & Run Type & Range \\
\hline A & 0 & $100 \leq Z \leq 999$ \\
\hline B & 0 & $10 \leq Z \leq 99$ \\
\hline C & 0 & $2 \leq Z \leq 9$ \\
\hline
\end{tabular}

\begin{tabular}{|c|c|c|}
\hline Symbol & Run Type & Range \\
\hline D & 1 & $100 \leq Z \leq 999$ \\
\hline E & 1 & $10 \leq Z \leq 99$ \\
\hline F & 1 & $2 \leq Z \leq 9$ \\
\hline
\end{tabular}

\section{EXPERIMENTAL RESULTS}

In this section the performances of the introduced compression algorithm is presented and compared to other known compression algorithms. For this purpose, data from the MIT-BIH arrhythmia database is used to evaluate the proposed compression algorithm. 
The obtained results are compared with that obtained using other well known ECG compression algorithms [1], [3], [14]-[16]. All ECG data used here are sampled at 360 $\mathrm{Hz}$, and the resolution of each sample is $11 \mathrm{bits} / \mathrm{sample}$. The distortion between the original and reconstructed signal is measured by percent root mean square difference $\left(\mathrm{PRD}\right.$ or $\left.\mathrm{PRD}_{1}\right)$. Although PRD does not exactly correspond to the result of a clinical subjective test, it is easy to calculate and compare, so it is widely used in the ECG data compression literature. The first two minutes of records 117 and 119 from MIT-BIH database were used to experimentally assess the performance of the method. We applied MDCT followed by DWT for each test signal and for target PRD equal to $1.5 \%, 2.0 \%, 2.5 \%$ and $3.0 \%$. The wavelets used for the assessment of the proposed method are: biorthogonal with 12/4 filters lengths, Daubechies $\mathrm{D}_{4}$, and symlets of order 4. The resulting WT-coefficients were then thresholded and coded using the above mentioned thresholding rule and coding technique respectively. Figure (1) allows visual assessment of the quality of four second length ECG signal from record 119. It indicates an excellent preservation of QRS complexes and of all important signal features.

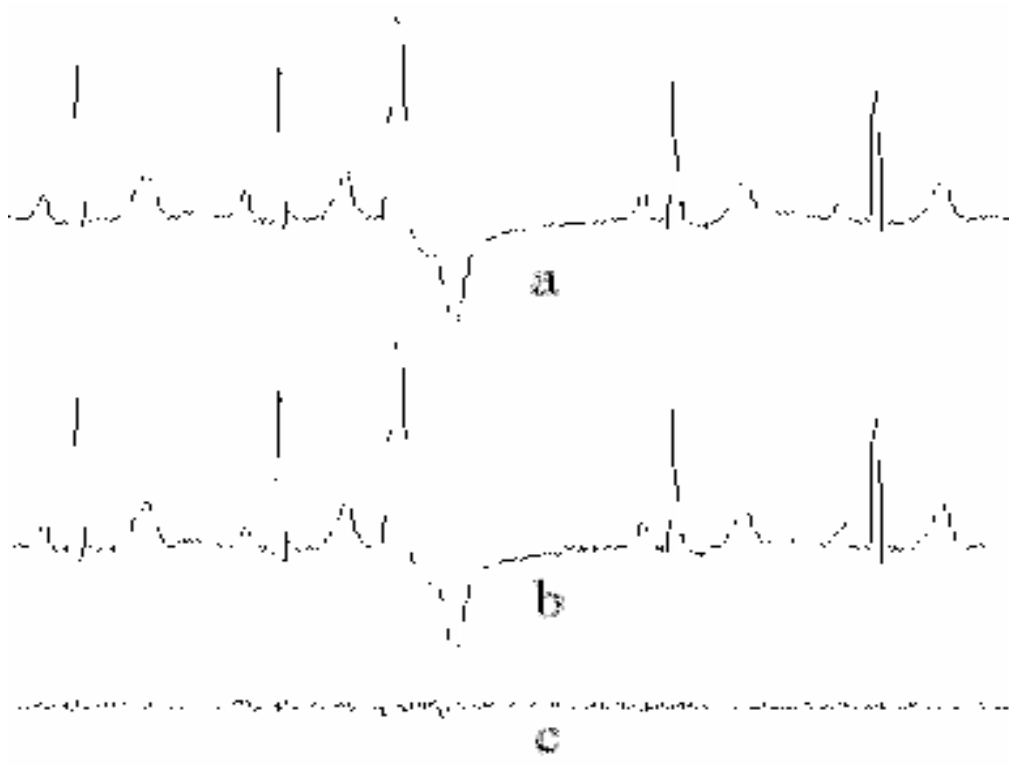

Fig. (1): Visual assessment of the quality of four second ECG signal from record 119.

(a) Original signal (b) Reconstructed signal (c) Error signal (with PRD=3.42\%).

In order to compare the performance of our method with other ECG compressors, we selected results from the literature reporting CR and PRD or $\mathrm{PRD}_{1}$ and obtained with precisely defined sections of records from the MIT/BIH arrhythmia database. Some of these results were produced after changing the sampling rate or the number of bits per sample of the signals, and we altered the signals accordingly. We compared PRD or $\mathrm{PRD}_{1}$ at the same CR, within a 0.04 tolerance. Table (2) summarizes the results. A compression ratio of 7.98:1 has been achieved with a percent of root mean square difference (PRD) of $0.25 \%$, indicating that the proposed compression technique offers 
the best performance over the other evaluated methods. For higher compression ratios up to $21.5: 1$, the PRDs are less than $5.89 \%$ which is acceptable from diagnoses point of view.

\section{CONCLUSION}

In this paper, we proposed a hybrid ECG compression technique based on wavelet transformation of the MDCT coefficients of the signal. While the DCT-based ECG coders perform very well at moderate bit rates, at higher compression ratios, ECG signal quality degrades. Wavelet-based coding on the other hand provides substantial improvement in signal quality at low bit rates because of overlapping basis functions and better energy compaction property of wavelet transforms. Interaction of MDCT analysis with data compression, DWT transformations, signals thresholding and coding are a few of the many outstanding challenges in ECG compression. We tested the performance of the algorithm by coding two records in MIT-BIH ECG arrhythmia database. Results obtained by running the compressor on the first two minutes of records 117 and 119 and adopting different MDCT and DWTs show that the proposed method is capable of achieving compressed ratio in between 12.6:1 and 21.5:1, with PRD less than $5.89 \%$ which would be suitable for most monitoring and diagnoses applications. We presented traces for visual assessment of the quality of the reconstructed signals. These traces indicate an excellent preservation of all important signal features.

Table (2): Comparing compressors from literature with the proposed compressor.

\begin{tabular}{|l|c|c|c|}
\hline \multicolumn{2}{|c|}{ Methods from Literature } & Proposed Method & \multirow{2}{*}{ CR } \\
\cline { 1 - 2 } Compression Method & $\begin{array}{c}\text { \% PRD or } \\
\text { \% PRD }\end{array}$ & $\begin{array}{c}\text { \% PRD or } \\
\% \text { PRD }_{1}(*)\end{array}$ & \\
\hline Wavelet Compression by SPIHT [1] & 3.0 & 2.5 & 10 \\
& 6.5 & 5.4 & 20 \\
\hline Sub-band/F16B FIR [14] & $2.8^{*}$ & $2.4^{*}$ & 7.3 \\
\hline Adaptive Optimized Quantization of & 6.8 & 6.0 & 12.5 \\
WT Coefficients [3] & 7.5 & 1.7 & 6.0 \\
Cut and Align Beats Approach with 1D & 15.1 & 4.0 & 12.0 \\
DCT [15] & $6.3^{*}$ & $3.4^{*}$ & 9.4 \\
Gold Washing VQ / WT [16] & $8.2^{*}$ & $4.7^{*}$ & 12.4 \\
\hline
\end{tabular}

\section{REFERENCES}

[1] Z. Lu, D. Kim, W. Pearlman, "Wavelet compression of ECG signals by set partitioning in hierarchical trees algorithm," IEEE Transactions on Biomedical Engineering, vol. 47, no. 7, pp. 849-856, 2000.

[2] S. M. S. Jalaleddine, C. G. Hutchens, R. D. Strattan, and W. A. Coberly, "ECG data compression techniques - a unified approach," IEEE Transactions on Biomedical Engineering, vol. 37, pp. 329-343, 1990. 
[3] J. Chen and S. Itoh, "A wavelet transform-based ECG compression method guaranteeing desired signal quality," IEEE Transactions on Biomedical Engineering, vol. 45, no. 12, pp. 1414-1419, 1998.

[4] H. Gholam-Hosseini, H. Nazeran, and B. Moran, "ECG compression: evaluation of FFT, DCT, and WT performance," Australasian-Physical-\&-EngineeringSciences-in-Medicine, vol. 21, no. 4, pp.186-92, Dec. 1998.

[5] V. Britanak and K. R. Rao, "A new fast algorithm for the unified forward and inverse MDCT/MDST computation," Signal Processing 82, 433-459, 2002.

[6] V. Nikolajevic and G. Fettweis, "A new Recursive Algorithm for the Unified Forward and Inverse MIDCT/MIDST," Journal of VLSI Signal Processing, vol. 9, no. 34, pp. 203-208, 2003.

[7] Che-Hong Chen, Bin-Da Liu, and Jar-Ferr Yang, "Recursive architectures for realizing modified discrete cosine transform and its inverse," IEEE Transactions Circuits Systems-II: Analog Digital Signal Processing, vol. 50, pp. 38-45, 2003.

[8] I. Daubechies, and C.A. Micchelli, "Ten lectures on wavelets," SIAM, 1994.

[9] M. Hilton, "Wavelet and wavelet packet compression of electrocardiograms," IEEE Transactions on Biomedical Engineering, vol. 44, pp. 394-402, 1997

[10] Sabah, M. Ahmed and M. Abo-Zahhad, " A new hybrid algorithm for ECG signal compression based on the wavelet transformation," Medical Engineering and Physics, vol. 24, no. 3, pp. 50-66, 2001.

[11] G. B Moody, "The MIT-BIH arrhythmia database CD-ROM," Second Ed., Harvard-MIT Division of Health Sciences and Technology, August 1992.

[12] A. Cohen, I. Daubechies, and J. C. Feauveau, "Biorthogonal bases of compactly supported wavelets," Comm. on Pure and Applied Mathematics, vol. XLV, pp. 485-560, 1992.

[13] Y. Zigel, A. Cohen, A. Kats, "The weighted diagnostic distortion (WDD) measure for ECG signal compression," IEEE Transactions on Biomedical Engineering, vol. 47, pp. 1422-1430, Nov. 2000.

[14] J. Husoy, and T. Gjerde, "Computationally efficient sub-band coding of ECG signals," Medical Engineering and Physics, vol. 18, no. 2, pp. 132-142, 1996.

[15] H. Lee, and K. Buckley, "ECG data compression using cut and align beats approach and 2-D transforms," IEEE Transactions on Biomedical Engineering, vol. 46, no. 5, pp. 556-564, 1999.

[16] S. Miaou, and H. Yen, "Quality driven gold washing adaptive vector quantization and its application to ECG data compression," IEEE Transactions on Biomedical Engineering, vol. 47, no. 2, pp. 209-218, 2000.

[17] M. Abo-Zahhad, and B. A. Rajoub, "ECG compression algorithm based on coding and energy compaction of the wavelet coefficients " The 8th IEEE International Conf. On Electronics, Circuits and Systems, Malta, pp. 441-444, Sept. 2001. 


\section{ضغط أشسارة القلب باستخدام محول جيب التمام المُعدَّل المتقطع ومحول المويجة المتقطع محن جيب}

د. صباح محمد أحمد

أستاذ المساعد بقسم الهندسة الكهربائيةِ ، كليّة الهندسة، جامعة اسيوط ، جمهورية مصر العربية (بريد الكتروني (sabahma@yahoo.com )

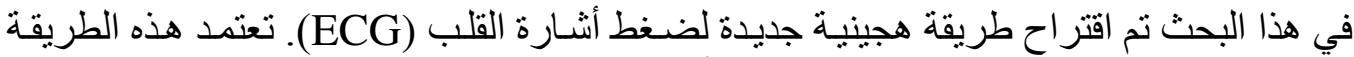

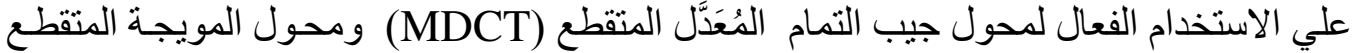

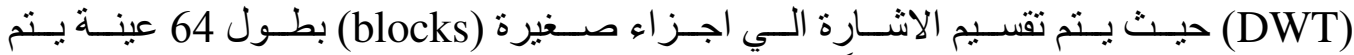

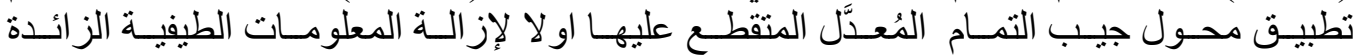

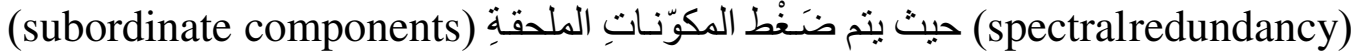

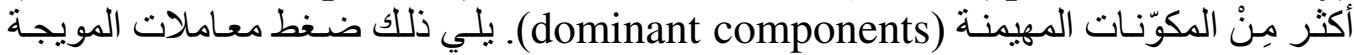

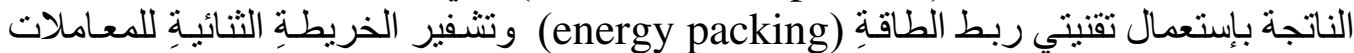

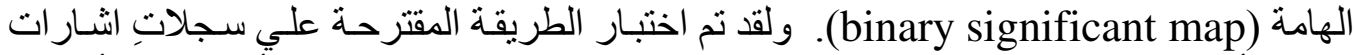

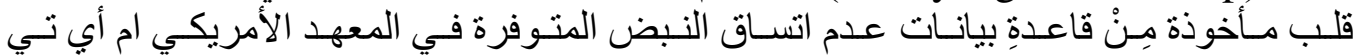

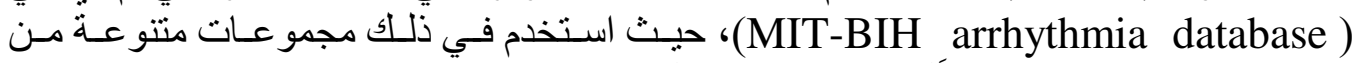

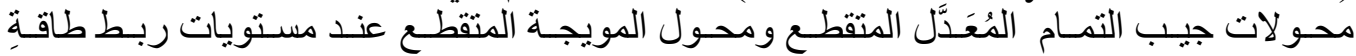

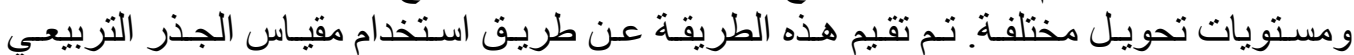

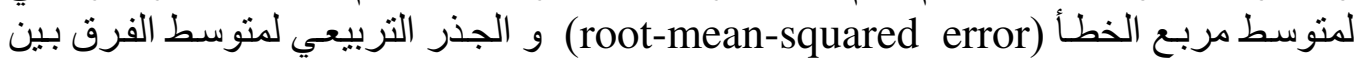

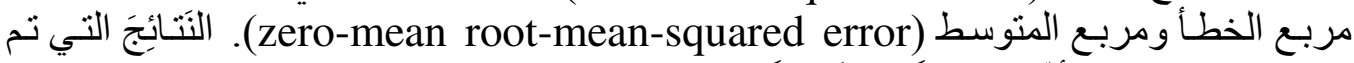

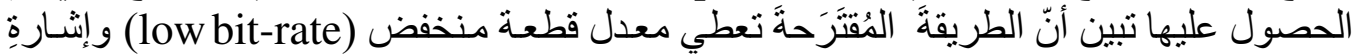

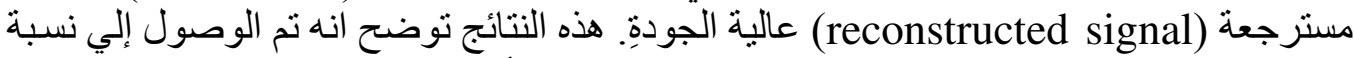

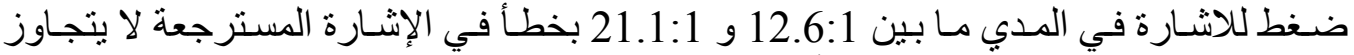

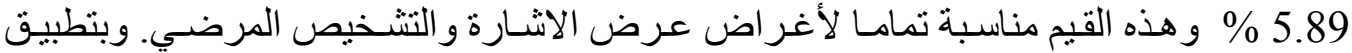

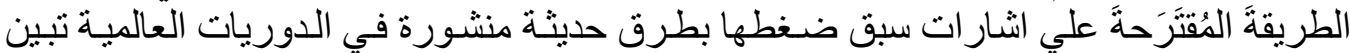
التكافئ الايجابي بينها. 